International Journal of Engineering \& Technology, $7(2.24)(2018) 299-303$
International Journal of Engineering \& Technology
SPC
Website: www.sciencepubco.com/index.php/IJET
Research paper

\title{
Leukaemia Detection in Microscopic Imagery using Optimization Algorithm
}

\author{
M.Venkata Dasu, P.Subbaiah* \\ Research Scholar, Dept of ECE, Rayalaseema University, Kurnool-518007, Andhra Pradesh \\ Dept of ECE, Nalla Narasimha Reddy Engineering College, Ghatkesar, Hyderabad-500088, Telangana \\ *Corresponding Author Email: subbaiah.nani45@gmail.com
}

\begin{abstract}
In this paper, automated approach of blood cancer detection is proposed. Usually microscopic images examined by experts manually are time consuming and less accuracy. The automated blood cancer detection system analyses the microscopic image and overcomes these drawbacks. The proposed system extracts the features of the image and applies filtering techniques. In this paper proposed method is cuckoo search optimization algorithm which is used in line with segmentation. The features of segmented image can be obtained from Scale invariant feature transform. Some of the features like PSNR, sensitivity, accuracy, etc. are calculated for leukemia detection. The performance is compared with the existing method ACO. The proposed system is tested on image dataset and $94.24 \%$ accuracy is achieved. The proposed system is successfully implemented in MATLAB.
\end{abstract}

Keywords: Leukemia CLAHE,Segmentation,Cuckoo Search(CSO),Ant Colony optimization(ACO),SIFT.

\section{Introduction}

Leukemia is one type of blood cancer. It is caused due to the uncontrollable growth of white blood cells and decreases in neutrophils cells count. Various methods are used to diagnosis Leukemia. Generally the Bone marrow smear images are composed of the various complex items. The proliferation and maturation of blood cells occur in the marrow only. It is known that the blood cells in a marrow smear can have different sizes and shapes [1-2]. To study the irregularities and to distinguish cell classification a greatly skilled resources are needed.

One of the traditional methods to detect the existence of blast cells that causes Leukemia can be attained through the microscopic observation by the hematologists in medical research. This method of exanimating the existence of leukemia through traditional microscopic observation is very time consuming, costly and monotonous. At present these methods to investigate the Leukemia do not fulfill the exact requirements. Hence a new approach is very essential which is free from the influence of the system operator fatigue. There is a need of cost effective and robust computer aided system which improves the reliability and efficiency of the leukemia diagnosis [3]. Now a day's digital image processing techniques are implemented in automated systems for medical diagnosis. Digital image processing affords an opportunity to extract significant and treasured information from an image. Digital Image processing technique is used to detect cancer in white blood cells (WBC) and it is also implemented to characterize the behavior of blood cell nucleus. DIP is a novel approach in identifying the cancer as it is cost and time effective in comparison to traditional microscopic method. For an affective detection of cancer through DIP technique, the basic and key requirement is to segment the WBC in systematic manner. Literature on Image segmentation is available. [4]. An efficient and quiet approachable method for WBC and their nucleus segmentation is the Edge detection technique [5][6].
In general an image is a composition of RGB colours. It finds difficult to process this composed RGB image. Hence a color conversion is needed for pre-processing of the image. In this connection, various color space conversion is used for segmenting white, red blood cells and the platelets. [7][8]. Traditionally there are several segmentation methods like Adaptive thresholding, Otsu segmentation, $\mathrm{K}$ means Clustering etc..

From the literature review it is observed that the segmentation accuracy of the traditional methods is less. To improve the segmentation accuracy optimization techniques are used for detection of cancer in white blood cells. This paper introduces a novel segmentation method named Cuckoo Search (CSO) optimization algorithm to segment the blast cells in microscopic images.

This Paper is structured as follows. Section-2 depicts the proposed method whose subsections discussed about image pre-processing method, Cuckoo search algorithm for segmentation of microscopic image, SIFT (Scale Invariant Feature Transform) for feature extraction and attribute calculation for segmented cell. Section-3 depicts the statistical comparison of obtained results from Ant colony optimization and Cuckoo search optimization. Finally, Section-4 presented conclusion.

\section{Methodology}

The main intension of the proposed work is to fragment the WBC in cancer (leukemia) and normal condition from the acquired stained peripheral blood film of microscopic image. The interconnection among WBCs is different in these two conditions where they are isolated from each other in course of normal condition and due to existence of blast cell they are connected in course of leukemia. Hence, a novel algorithm has been evolved such that it separates WBCs during leukemia and does not obstruct the effect of separation process during normal condition. In proposed method the noise is removed by median filter and RGB microscopy image is converted in to grayscale conversion 
and the segmentation is applied to detect the malignant cell in microscopy images. Fig. 1 shows the segmentation of WBC Nucleus by proposed Algorithm.

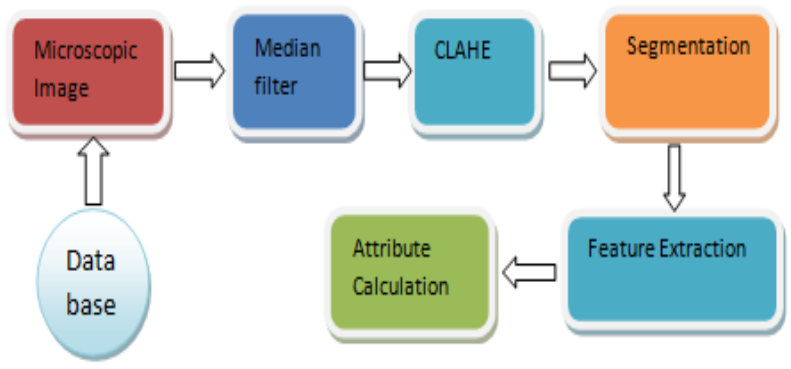

Fig.1: Block diagram of proposed model

\subsection{Image Acquisition}

The Initial step in processing of image to segment WBC is the image acquisition. Microscopic images are acquired from public database.

\subsection{Pre-processing}

Typically the acquired images are in the RGB color space from digital microscopes. This is slightly tedious and critical to segment. Depends on some factors such as settings of camera, aging stain and inconsistent illumination, the color and intensity of background and blood cells are differs slightly. In order to guard the cell segmentation the input image which is in RGB is converted into gray scale image. Generally a microscopic image contains low frequency noise this can be removed by median filter and then CLAHE method is applied to improve the contrast of an image.

- Median filter: It is a nonlinear digital filter used to remove noise. It operates on input image where it finds out the median value of a sub image [9]. Let the pixels of an image be an odd number $\mathrm{P}$ then the pixels of sub image is written as $\mathrm{Y}=\left[\mathrm{Y}_{\mathrm{l}}\right.$, ..., $\left.\mathrm{Y}_{\mathrm{P}}\right]$. The output of median filter output is the $(\mathrm{P}+1) / 2$

$$
\text { Median of }(\mathrm{Y})=\mathrm{Y}(\mathrm{P}+1) / 2
$$

- CLAHE: It is a contrast enhancement technique which is well suited for enhancement of microscopic images. It removes noise and enhances the quality of an image also avert the over amplification of noise [10]. This technique is differs from normal adaptive histogram equalization methods. The feature of CLAHE can also be extended to global histogram equalization method which yields to contrast limited histogram equalization (CLAHE). CLAHE derives the transformation function by applying the procedure of contrast limiting to individual neighborhood pixels.

\subsection{Segmentation}

Segmentation is essential in medical applications where to extract the significant information from a given input image. It plays a significant role for feature extraction and classification. In this paper, Cuckoo search optimization algorithm has been used. This algorithm is efficient when clustering large and high-dimensional databases.

\subsection{Scale Invariant Feature Transform (SIFT)}

Feature extraction is defined as "technique of redefining a large set of redundant data into a set of feature vectors of reduced dimension"[11]. In this paper, colors features are extracted from the nucleus using SIFT [12]. SIFT contains scale-space extreme detection, key point localization, orientation assignment and key point descriptor.
- Scale-space extreme detection: The primary step of computation searches comprises of image locations and overall scales.

- Key point localization: To find out location and scale an exhaustive model is established at each candidate location and based on the stability values key points are selected.

- Orientation assignment: For each and every key point location more than one orientation are allocated based on gradient directions of local image. The image data has been transformed based on scale, orientation, and location of each feature and all the required operations are performed on transformed image.

- Key point descriptor: At the selected scale region, each key point local image gradients are measured and these gradients are transformed in to a representation where considerable level of illumination is changed [13].

\section{Optimization Algorithms}

\subsection{Ant Colony Algorithm}

It is an evolutionary meta-heuristic simulation algorithm enthused by the real behavior of ants. It finds the shortest path between two locations. [14]. It is used to solve the optimization problems such as map coloring, pipeline placing, traveling salesman problem, workshop task scheduling etc. This algorithm is applicable to data cluster methods and also has a wide application in view of fuzzy clustering methods [15]. The major drawbacks of ACO are local search and poor performance. Hence an effective cuckoo search optimization is proposed to perform the local search and increases the performance.

\subsection{Cuckoo Search Algorithm}

It is a nature inspired and efficient optimization method invented in the year 2009 by Yang and Deb [16]. In Cuckoo search algorithm based on the location of eggs laid in host form the feasible solutions are encoded where as in other inspired algorithms the individuals travel in the search space. The algorithm initiates with a population size. For an $\mathrm{n}$ host nests which are randomly placed with dimension $\mathrm{k}$ is written as

$\mathrm{Y}_{\mathrm{i}}=\left(\mathrm{Y}_{\mathrm{i} 1}, \mathrm{Yi}_{2} \ldots . . . \mathrm{Y}_{\mathrm{ik}}\right)$

Where $\mathrm{k}$ is the problem domain dimension.

In evolution loop at each step the levy flights generates a new solutions and is evaluated. Based on the evaluated value it restores with another better randomly chosen solution [17]. In the invention step a part of host forms are deserted and new nests are formed in arbitrary positions. Finally the best solution is retaining based on the solutions estimated at end of each loop. CSA also solve definite optimization problems due to presence of larger better variants [18]

\section{Results and Discussion}

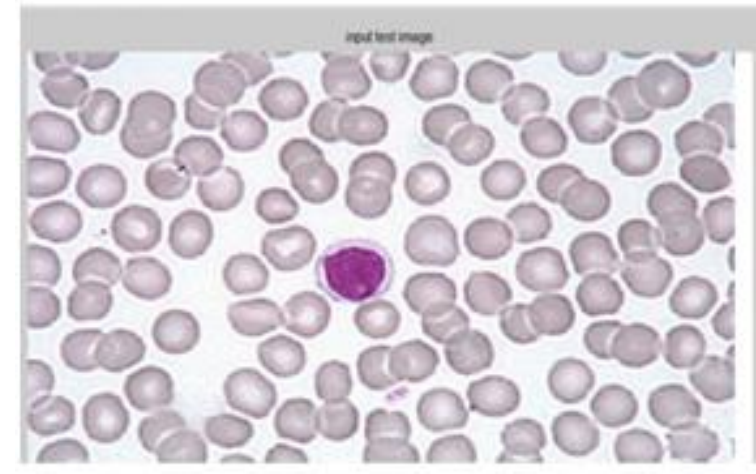

Fig. 1: Cancerous microscopic RGB image. 
The input image is shown Fig. 1 in which is acquired from open source medical image database (Courtesy: Nikon Microscopyu). This has a malignant white blood cell which is shown up with bold purple color. In cancerous blood smear the size of cells are large and have variable size of nucleus. Generally microscopic images acquired are available in RGB format. The direct processing of the image may lead to distortions and improper results. Thus this image is converted into gray shade image where the intensity levels are single when compared to RGB

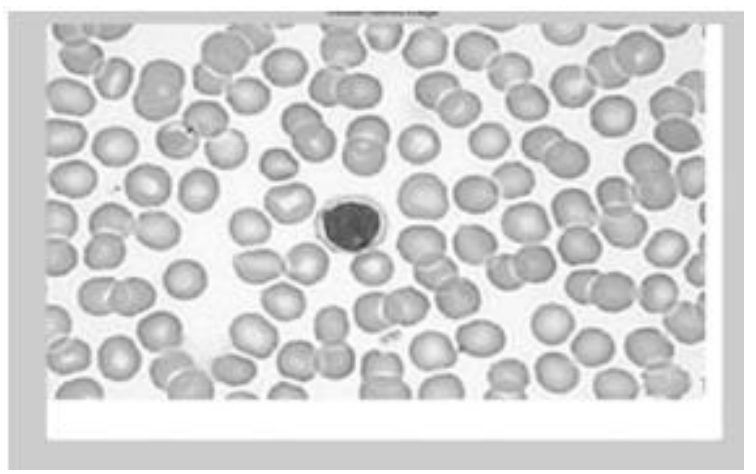

Fig. 2: Median Filtered image.

Fig. 2 represents the Median Filtered image. As the acquired images contain noise added due to various factors it is essential to involve the filters. Thus in the proposed model median filter is used to reduce noise.

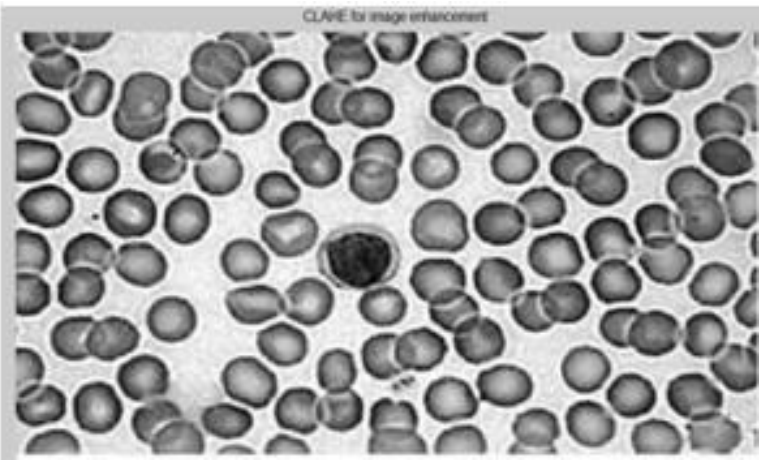

Fig. 3: CLAHE image.

Fig. 3 represents CLAHE image. It is a contrast enhancement technique which reduces the over amplification of noise. CLAHE will make the edges of the white blood cells sharper in the image

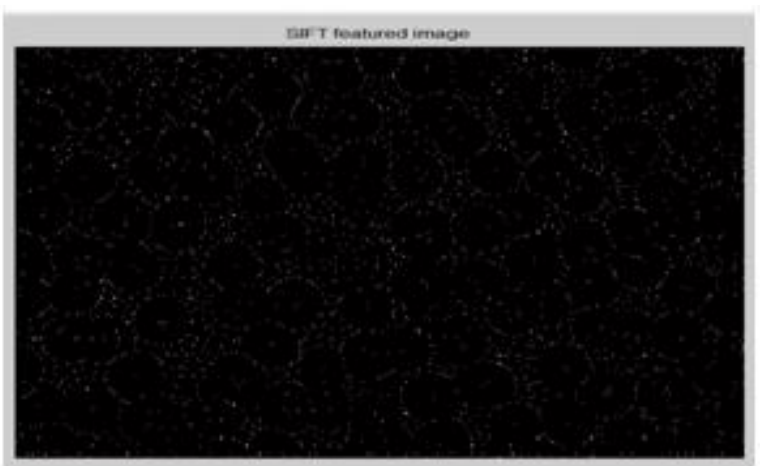

Fig. 4: SIFT Featured image

In order determine the boundaries and feature extraction as shown in figure 4 Scale invariant feature transform (SIFT) method, by dimensionality reduction. The input data will be transformed into a reduced representation set of features when the input data applied to an algorithm is large. Transforming the input data into the set of features is called feature extraction, which is used to extract the individual cells from the input image.

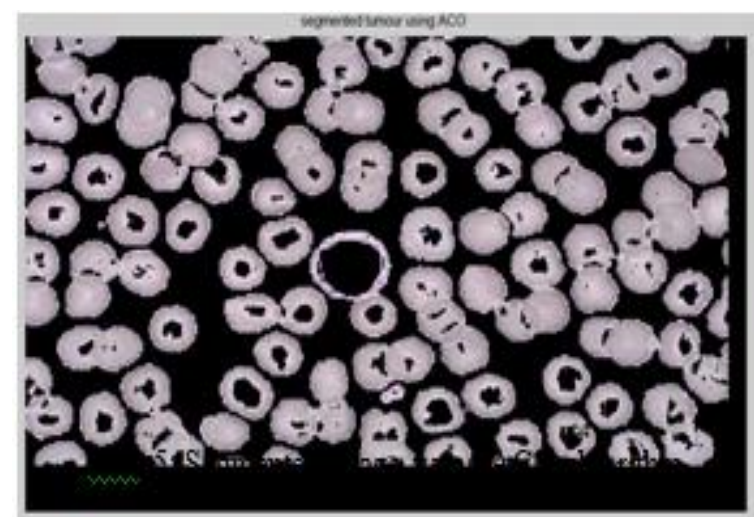

Fig. 5: Segmented image using ACO algorithm

The image in Fig. 5 show the resulted images after segmentation process based on ACO algorithm. It is obvious from the Fig.5 that affected cell is in differentiable from healthy ones. Detection of abnormal part is difficult with ACO.

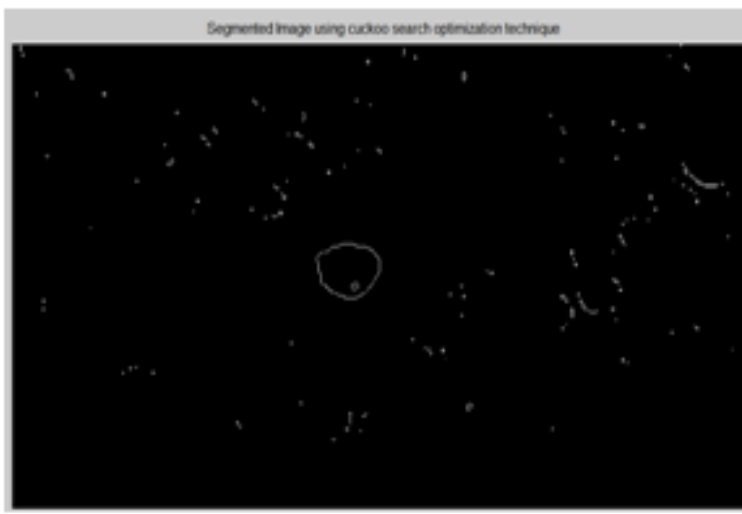

Fig. 6: Segmented image using CSO algorithm

The image in Fig.6 shows the resulted images after segmentation process based on CSO algorithm. Where it is very clear that only abnormal cell is derived and shown with exact boundary.

\section{Objective Analysis:}

Once the subjective analysis is carried out as shown in previous section it is obligatory to analyze it with the respective image attributes obtained for both ACO and Cuckoo search algorithms. This is referred as objective analysis which is represented in Table1.

Table 1: Performance Measures

\begin{tabular}{|c|l|c|c|}
\hline S.No & Parameter & ACO & $\begin{array}{c}\text { Cuckoo } \\
\text { Search }\end{array}$ \\
\hline 1 & PSNR & 30.61 & 31.08 \\
\hline 2 & Accuracy & 92.80 & 93.20 \\
\hline 3 & Sensitivity & 80.05 & 82.32 \\
\hline 4 & CPU time(Sec) & 11 & 09 \\
\hline
\end{tabular}

After an experimental investigation it is shown that Peak Signal to Noise Ratio (PSNR), accuracy, Sensitivity and CPU time are better for Cuckoo search algorithms. The same can be represented as a graphical representation which is shown in figure 7 where it is easily perceivable that how Cuckoo is better in all aspects. 


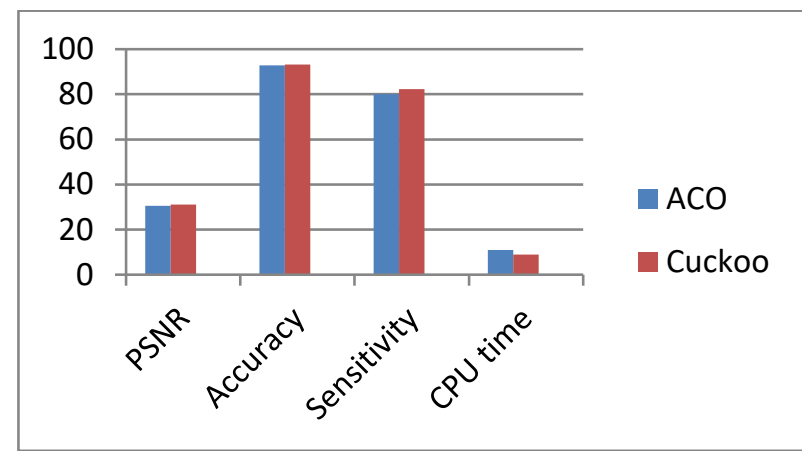

Fig. 7: Graphical representation of parameters

\section{Conclusions}

The proposed Cuckoo search optimization algorithm is used to detect Leukaemia from microscopic images of blood samples. Here the investigation is carried out for existing ACO and proposed Cuckoo search optimization algorithms. The proposed system has efficient, reliable, high segmentation accuracy and less CPU time over existing method.

\section{Acknowledgment}

The authors are thankful to Annamacharya Institute of Technology \& Sciences, Rajampet and Rayalaseema university, Kurnool for their extensive support in carrying this research work.

\section{References}

[1] A. Bell, S. Sallah, Morphology of Human Blood Cells. USA: Abbott Diagnostic,2005.

[2] P. Mittal, K.R. Meehan. The Acute Leukemia, Clinical Review Article, Hospital Physician, pp 37-44.

[3] Huey Nee Lim(2015).Color and Morphological Based Techniques on White Blood Cells Segmentation in International Conference on Biomedical Engineering,2001.

[4] Rakibull Ahasan et.al. White Blood Cells Nucleus Segmentation from Microscopic Images of strained peripheral blood film during Leukemia and Normal Condition. 5th International Conference on Informatics, Electronics and Vision, 2016, pp.361-366.

[5] ] Jha, K.K.; Das, B.K.; Dutta, H.S., "Detection of abnormal blood cells on the basis of nucleus shape and counting of WBC," in Green Computing Communication and Electrical Engineering (ICGCCEE), vol., no., pp.1-5, 6-8 March 2014.

[6] Hari, J.; Prasad, A.S.; Rao, S.K., "Separation and counting of blood cells using geometrical features and distance transformed watershed," in Devices, Circuits and Systems (ICDCS), pp.1-5, 6-8 March 2014.

[7] Fahimuddin Shaik, Anil Kumar Sharma, Syed Musthak Ahmed, "Hybrid Model for Analysis of Abnormalities in Diabetic Cardiomyopathy and Diabetic Retinopathy related images", Springer Plus Journal, Springer Publications, April 2016

[8] Tulsani, H.; Gupta, R.; Kapoor, R., "An improved methodology for blood cell counting," in Multimedia, Signal Processing and Communication Technologies (IMPACT), pp.88-92,23- 25 Nov. 2013

[9] Sharif, J.M.; Miswan, M.F.; Ngadi, M.A.; Salam, M.S.H Mahadi bin Abdul Jamil, M., "Red blood cell segmentation using masking and watershed algorithm: A preliminary study," in Biomedical Engineering (ICoBE),pp.258-262, 27-28 Feb. 2012.

[10] Markus Beermann, Adeel Jalil and Jens-Rainer Ohm, "Thresholded Weighted Median Filters for Ringing Reduction in Processed Images",2005.

[11] Nabin Kharel, Abeer Alsadoon, P.W.C. Prasad, A. Elchouemi," Early Diagnosis of Breast Cancer Using Contrast Limited Adaptive Histogram Equalization (CLAHE) and Morphology Methods", 8th International Conference on Information and Communication Systems (ICICS), pp.120-124, 2017.

[12] Mohapatra S, Patra D. Automated Cell Nucleus Segmentation and Acute Leukemia Detection in Blood Microscopic Images.
2010 IEEE International Conference on Systems in Medicine and Biology (ICSMB) 2010:4954

[13] Preetham Kumar, Shazad Maneck," Automatic Detection of Acute Myeloid Leukemia from Microscopic Blood Smear Image" IEEE,pp 1803-1807,2017.

[14] Jing Xu, Gongliu Yang, Yuanyuan Liu, Jingiia Zhong," Coin Recognition Method based on SIFT Algorithm" 4th International Conference on Information Science and Control Engineering, pp 229-233, 2017.

[15] Lei Li, Yuemei Ren, Xiangpu Gong,” Medical Image Segmentation Based on Modified Ant Colony Algorithm with GVF Snake Model", International Seminar on Future Biomedical Information Engineering, pp11-14,2008.

[16] Qidi Wu, Lei Wang, "Intelligent ant algorithm and application", Shanghai Science and Technology Education Press, Shanghai, 2004.

[17] X.-S. Yang, "Nature-Inspired Optimization Algorithms", Elsevier Inc., 2014.

[18] S.I. Bejinariu, H. Costin, F. Rotaru, R. Luca, C. Nita, "Automatic Multi-threshold Image Segmentation Using Metaheuristic Algorithms", International Symposium on Signals, Circuits and Systems, ISSCS 2015, Iasi, Romania, 2015.

[19] Silviu-Ioan Bejinariu, Hariton Costin, Florin Rotaru1, Ramona Luca, Cristina Nita," Image Processing by means of Some BioInspired Optimization Algorithms", The 5th IEEE International Conference on E- Health and Bioengineering - EHB 2015

[20] Avinash Yadlapati, Dr. Hari Kishore Kakarla, "An Advanced AXI Protocol Verification using Verilog HDL", Wulfenia Journal, ISSN: 1561-882X, Volume 22, Number 4, pp. 307-314, April 2015

[21] P Ramakrishna, K. Hari Kishore, "Design of Low Power 10GS/s 6-Bit DAC using CMOS Technology "International Journal of Engineering and Technology(UAE), ISSN No: 2227-524X, Vol No: 7, Issue No: 1.5, Page No: 226-229, January 2018.

[22] A Murali, K. Hari Kishore, "Efficient and High Speed Key Independent AES Based Authenticated Encryption Architecture using FPGAs "International Journal of Engineering and Technology(UAE), ISSN No: 2227-524X, Vol No: 7, Issue No: 1.5, Page No: 230-233, January 2018.

[23] G.S.Spandana, K Hari Kishore "A Contemporary Approach For Fault Diagnosis In Testable Reversible Circuits By Employing The CNT Gate Library" International Journal of Pure and Applied Mathematics, ISSN No: 1314-3395, Vol No: 115, Issue No: 7, Page No: 537-542, September 2017.

[24] K Hari Kishore, CVRN Aswin Kumar, T Vijay Srinivas, GV Govardhan, Ch Naga Pavan Kumar, R Venkatesh "Design and Analysis of High Efficient UART on Spartran-6 and Virtex-7 Devices", International Journal of Applied Engineering Research, ISSN 0973-4562, Volume 10, Number 09 , pp. $23043-$ 23052, June 2015

[25] K Bindu Bhargavi, K Hari Kishore "Low Power BIST on Memory Interface Logic", International Journal of Applied Engineering Research, ISSN 0973-4562, Volume 10, Number 08 ,pp. 21079-21090, May 2015

[26] Korraprolu Brahma Reddy, K Hari Kishore, “A Mixed Approach for Power Dissipation Reduction in Nanometer CMOS VLSI circuits", International Journal of Applied Engineering Research, ISSN 0973-4562 Volume 9, Number 18, pp. 5141-5148, July 2014.

[27] Nidamanuri Sai Charan, Kakarla Hari Kishore "Reorganization of Delay Faults in Cluster Based FPGA Using BIST" Indian Journal of Science and Technology, ISSN No: 0974-6846, Vol No.9, Issue No.28, page: 1-7, July 2016.

[28] Sravya Kante, Hari Kishore Kakarla, Avinash Yadlapati,"Design and Verification of AMBA AHB-Lite protocol using Verilog HDL" International Journal of Engineering and Technology, EISSN No: 0975-4024, Vol No.8, Issue No.2, Page:734-741, May 2016.

[29] Bandlamoodi Sravani, K Hari Kishore, "An FPGA Implementation of Phase Locked Loop (PLL)", International Journal of Applied Engineering Research, ISSN 0973-4562, Volume 10, Number 14,pp. 34137-34139, August 2015

[30] Avinash Yadlapati, Kakarla Hari Kishore,"Constrained Level Validation of Serial Peripheral Interface Protocol", Proceedings of the First International Conference on SCI 2016, Volume 1, Smart Computing and Informatics, Smart Innovation, Systems and Technologies 77, ISSN No: 2190-3018, ISBN: 978-981-105544-7, Chapter No: 77, pp. 743-753, 25 $5^{\text {th }}$ December 2017.

[31] P Kiran Kumar, P Prasad Rao, Kakarla Hari Kishore, "Optima Design of Reversible Parity Preserving New Full Adder / Full 
Subtractor", IEEE SPONSORED 3rd INTERNATIONAL

CONFERENCE ON ELECTRONICS AND COMMUNICATION SYSTEMS (ICECS 2016), pp. 3465-3470, $25^{\text {th }}$ and $26^{\text {th }}$ February 2016

[32] Y Avinash, K Hari Kishore ''Designing Asynchronous FIFO for Low Power DFT Implementation' International Journal of Pure and Applied Mathematics, ISSN No: 1314-3395, Vol No: 115, Issue No: 8, Page No: 561-566, September 2017

[33] Mahesh Mudavath and K Hari Kishore "Design of RF Front End CMOS Cascade CS Low Noise Amplifier on 65nm Technology Process" International Journal of Pure and Applied Mathematics, ISSN No: 1314-3395, Vol No: 115, Issue No: 7, Page No: $417-$ 422, September 2017.

[34] P. Sahithi K Hari Kishore, E Raghuveera, P. Gopi Krishna "DESIGN OF VOLTAGE LEVEL SHIFTER FOR POWEREFFICIENT APPLICATIONS USING 45nm TECHNOLOGY" International Journal of Engineering and Technology(UAE), ISSN No: 2227-524X, Vol No: 7, Issue No: 2.8, Page No: 103 108, March 2018.

[35] N Bala Dastagiri K Hari Kishore "A 14-bit 10kS/s Power Efficient $65 \mathrm{~nm}$ SAR ADC for Cardiac Implantable Medical Devices" International Journal of Engineering and Technology(UAE), ISSN No: 2227-524X, Vol No: 7, Issue No: 2.8, Page No: 34-39, March 2018.

[36] Meka Bharadwaj, Hari Kishore "Enhanced Launch-Off-Capture Testing Using BIST Designs" Journal of Engineering and Applied Sciences, ISSN No: 1816-949X, Vol No.12, Issue No.3, page: 636-643, April 2017.

[37] P Bala Gopal, K Hari Kishore, R.R Kalyan Venkatesh, P Harinath Mandalapu "An FPGA Implementation of On Chip UART Testing with BIST Techniques", International Journal of Applied Engineering Research, ISSN 0973-4562, Volume 10, Number 14, pp. 34047-34051, August 2015.

[38] S.V. Manikanthan , T. Padmapriya "An enhanced distributed evolved node-b architecture in 5G tele-communications network" International Journal of Engineering \& Technology (UAE), Vol 7 Issues No (2.8) (2018) 248-254.March2018

[39] S.V. Manikanthan, T. Padmapriya, Relay Based Architecture For Energy Perceptive For Mobile Adhoc Networks, Advances and Applications in Mathematical Sciences, Volume 17, Issue 1, November 2017, Pages 165-179

[40] S.V.Manikanthan and T.Padmapriya "Recent Trends In M2m Communications In 4g Networks And Evolution Towards 5g", International Journal of Pure and Applied Mathematics, ISSN NO:1314-3395, Vol-115, Issue -8, Sep 2017. 